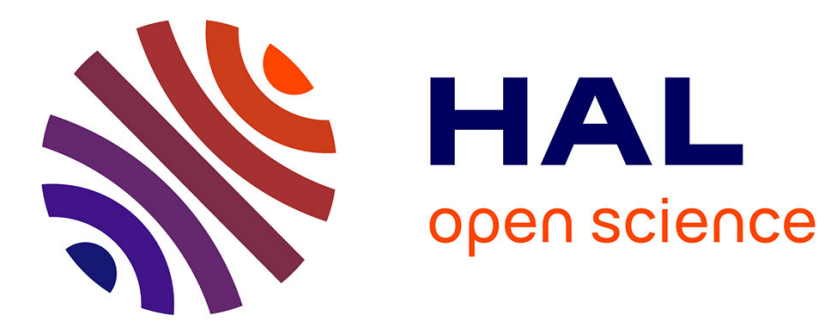

\title{
Les sécrétions exocrines des hétéroptères (allomones et phéromones). Une mise au point bibliographique \\ Claude Pavis
}

\section{To cite this version:}

Claude Pavis. Les sécrétions exocrines des hétéroptères (allomones et phéromones). Une mise au point bibliographique. Agronomie, 1987, 7 (8), pp.547-561. hal-00885026

\section{HAL Id: hal-00885026 \\ https://hal.science/hal-00885026}

Submitted on 1 Jan 1987

HAL is a multi-disciplinary open access archive for the deposit and dissemination of scientific research documents, whether they are published or not. The documents may come from teaching and research institutions in France or abroad, or from public or private research centers.
L'archive ouverte pluridisciplinaire HAL, est destinée au dépôt et à la diffusion de documents scientifiques de niveau recherche, publiés ou non, émanant des établissements d'enseignement et de recherche français ou étrangers, des laboratoires publics ou privés. 


\title{
Les sécrétions exocrines des hétéroptères (allo- mones et phéromones). Une mise au point bibliographique
}

\author{
Claude PAVIS \\ I.N.R.A., Laboratoire des Médiateurs chimiques, domaine de Brouëssy, Magny-les-Hameaux, F 78470 Saint- \\ Rémy-lès-Chevreuse
}

RÉSUMÉ

\begin{abstract}
Cet exposé est une revue des connaissances actuelles sur les sécrétions des Hétéroptères pouvant jouer un rôle dans la communication chimique interspécifique ou intraspécifique. Les différents types de glandes productrices de ces sécrétions sont décrits ainsi que leur mode de fonctionnement, la nature chimique des substances émises et leur rôle lorsqu'il est connu. Il ressort que ces sécrétions sont très diverses et peuvent se comporter comme

- des allomones (défense contre des prédateurs ou des agents pathogènes),

- des kairomones (attraction de certains parasites),

- des phéromones (agrégatives, aphrodisiaques ou sexuelles).
\end{abstract}

Mots clés additionnels : Glandes exocrines, substances de défense, kairomones.

\begin{abstract}
Exocrine secretions in Heteroptera (allomones and pheromones). A review.
This paper reviews present knowledge concerning the role played by chemical signals in both adults and nymphs of Heteroptera. We describe the different glands, their function and the chemical structure of the compounds emitted by the bugs. This study revealed that the different secretions may act as : allomones (defense against predators and microorganisms) ; kairomones (attraction of parasitoids) ; pheromones (aggregation, aphrodisiac or sex pheromones).
\end{abstract}

Additional key words : Exocrine glands, defensive secretions, kairomones.

\section{INTRODUCTION}

Les représentants de l'ordre des Hétéroptères sont particulièrement riches en glandes tégumentaires de toutes sortes. Elles ont été largement étudiées, particulièrement celles produisant les sécrétions dites défensives chez les adultes et les larves. Outre ces substances dont la nature et le rôle seront discutés, nous nous sommes intéressés aux sécrétions jouant un rôle dans la communication chimique intraspécifique.

Quelques cas sont élucidés tant au niveau des glandes productrices que de l'activité biologique et de la structure des phéromones ; cependant, la plupart des informations concernant ce sujet restent fragmentaires et parfois purement hypothétiques, c'est pourquoi il nous a paru important de préciser l'état actuel de nos connaissances dans ce domaine.

\section{GLANDES ODORIFIQUES}

Ce sont des glandes situées dans diverses parties du corps des jeunes et des adultes et qui émettent leurs sécrétions en réponse à une perturbation de l'insecte. Les auteurs ont longtemps pensé que leur seule action était de se protéger ou de faire fuir les agresseurs ; nous verrons que ce n'est pas toujours le cas.

Nous avons distingué le cas des jeunes de celui des adultes car il existe entre eux des différences sensibles quant à la structure productrice et à la nature des sécrétions, ce qui est peut-être le reflet de différences fonctionnelles.

\section{A. Les glandes des larves}

\section{Position et nombre}

Chez les larves d'Hétéroptères, ces glandes sont médianes et situées dorsalement au niveau de la membrane intersegmentaire des segments abdominaux. Leur nombre primitif est de quatre. C'est encore le cas au sein de la famille des Cryptostemmatidae (CARAYON, 1948). Souvent, la plus antérieure des glandes a une fonction ou des fonctions différentes de 
celles des glandes postérieures, ce qui peut se traduire par des différences qui apparaissent lors de l'ontogenèse.

$\mathrm{Au}$ cours de l'évolution, on assiste à une régression du nombre de ces glandes; le plus souvent, on en observe 3, parfois moins. Chez beaucoup de Pentatomidae (ALDRICH et al., 1978b), elles sont localisées entre les tergites abdominaux III-IV, IV-V et V-VI. Chez Oncopeltus fasciatus Dal. (Lygaeidae), il n'y a que 2 glandes, la plus antérieure ayant disparu.

\section{Fonctionnement}

En réponse à une perturbation ou à une agression, une partie du contenu des glandes est émis par l'insecte et se répand de diverses manières selon les groupes considérés.

Chez les jeunes Pentatomidae, Anthocoridae et Coreidae on observe parfois une projection de la sécrétion de façon unilatérale dans la direction d'où vient le danger potentiel (REMOLD, 1963). Par contre, quelques larves de Lygaeidae (Nysus spp., Henestaris sp.) font simplement couler leur sécrétion sur la face dorsale de leur abdomen aux alentours de l'orifice de sortie. Certains jeunes Pyrrhocoridae et Lygaeidae (dont $O$. fasciatus) humectent leurs tarses médians et postérieurs avec la sécrétion en les plaçant et les remuant au niveau de l'orifice de sortie ; ils "brossent " ensuite leur agresseur avec cet instrument empoisonné (OLAGBEMIRO \& STADDON, 1983).

Le fonctionnement de ces glandes est donc indépendant puisque dans la plupart des cas, seule celle située du côté de l'agresseur se décharge. De plus, on observe également des différences de fonctionnement entre les glandes antérieures et postérieures; chez $O$. fasciatus, les jeunes déchargent toujours leur réservoir postérieur en premier, un stress plus violent est nécessaire pour que la glande antérieure se décharge à son tour. On constate le même phénomène chez Nezara viridula L. (observation personnelle).

\section{Structure (fig. 1)}

Ces glandes dorso-abdominales sont formées d'un repli interne de la membrane intersegmentaire, non évaginable. La lumière du sac est donc bordée de cuticule. Les cellules glandulaires forment le plus souvent et primitivement un revêtement continu mais tendent au cours de l'évolution à s'isoler pour former 2 sacs latéraux de part et d'autre d'un réservoir médian dépourvu de cellules glandulaires, au moins en ce qui concerne la glande antérieure.

\section{Contenu}

La structure chimique des différentes substances identifiées chez les larves d'Hétéroptères est indiquée dans le tableau 1.

La plupart des travaux d'analyse structurale effectués sur le contenu des glandes dorso-abdominales l'ont été de façon globale, sans tenir compte de différences éventuelles entre glandes antérieures et postérieures; quelques auteurs ont cependant noté des différences.
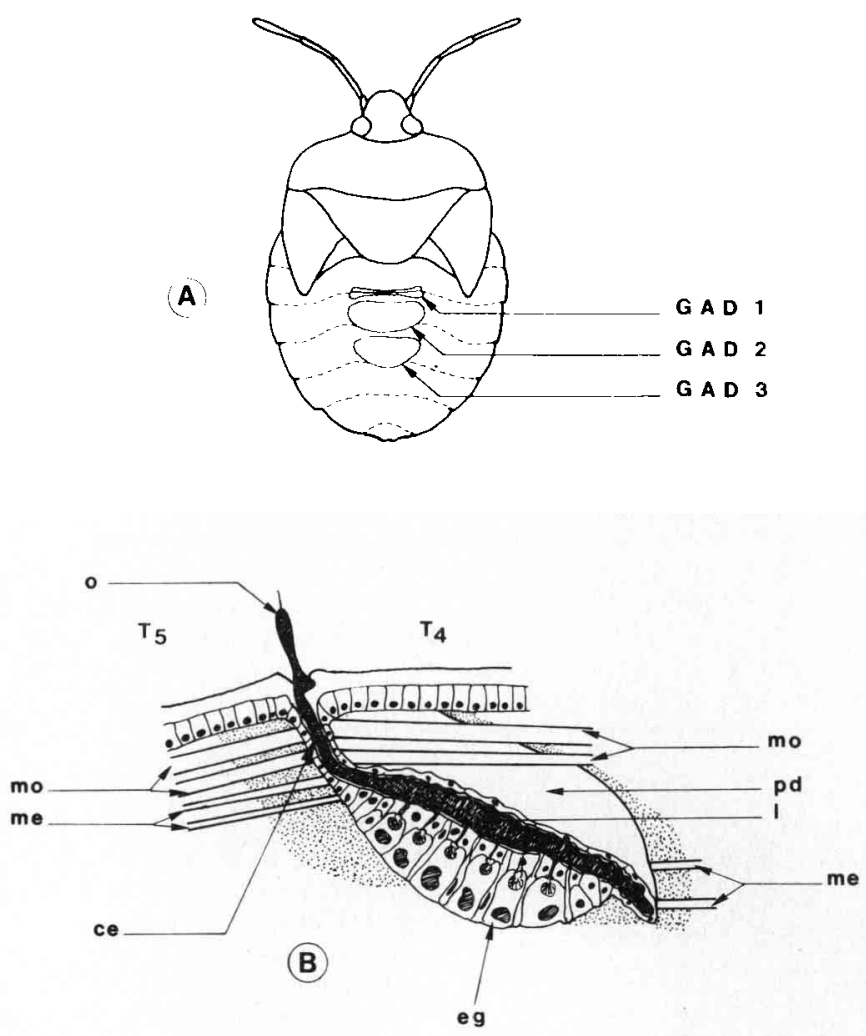

Figure 1

Glandes abdominales dorsales des jeunes Hétéroptères. $A$ : débouchés des 3 glandes chez un Pentatomidae (impair pour la glande antérieure ( $G A D$ 1), pairs pour les glandes postérieures (GAD 2 et $G A D 3)$; $B$ : diagramme indiquant la structure de la $2^{e}$ glande abdominale dorsale chez Pyrrhocoris apterus (Pyrrhocoridae). T4 et $T 5: 4^{e}$ et $5^{e}$ tergites abdominaux; o : orifice de sortie des sécrétions; mo: muscles commandant l'ouverture du canal efférent; me : muscle élongateur du sac glandulaire; ce : canal efférent ; $p d$ : paroi dorsale du sac glandulaire; eg: épithélium glandulaire; l: lumière du sac glandulaire. D'après STADDON, 1979.

Dorsal abdominal glands in Heteropteran nymphs. $A$ : abdominal ostioles in a Pentatomid. The first ostiole is divided (GAD I) and the others are undivided (GAD 2, GAD 3). B : Diagram indicating the structure of the 2nd abdominal scent gland from Pyrrhocoris apterus (Pyrrhocoridae). T4 and T5: 4th and 5th abdominal tergites; $o:$ ostiole pore; mo: opener muscle of the abdominal scent gland; me: stretch muscle of the abdominal scent gland; ce : discharge canal; pd : dorsal wall of gland sac; eg : glandular epithelium; l: lumen of gland sac. From STADDON, 1979.

EVERTON et al. (1974) ont montré chez $O$. fasciatus que le réservoir de la $1^{\text {re }}$ glande contenait un liquide homogène et incolore tandis que celui de la $2^{\mathrm{c}}$ était empli d'une sécrétion formée de 2 phases, l'une incolore, l'autre jaune. Les composés présents dans ces 2 glandes sont des composés aliphatiques insaturés mais de nature différente. Ces différences de composition et de fonctionnement suggèrent 2 fonctions distinctes.

Chez Dysdercus intermedius Dist. (Pyrrhocoridae), la nature des sécrétions n'est pas non plus la même pour les 2 glandes (CALAM \& YouDEOWI, 1968).

De même, chez Nezara viridula L. (observations personnelles), une étude préliminaire en chromatographie en phase gazeuse montre que la plus antérieure des glandes produit des composés différents de ceux des glandes postérieures et ceci quel que soit le stade considéré.

GOUGH et al. (1985) ont analysé séparément le con- 


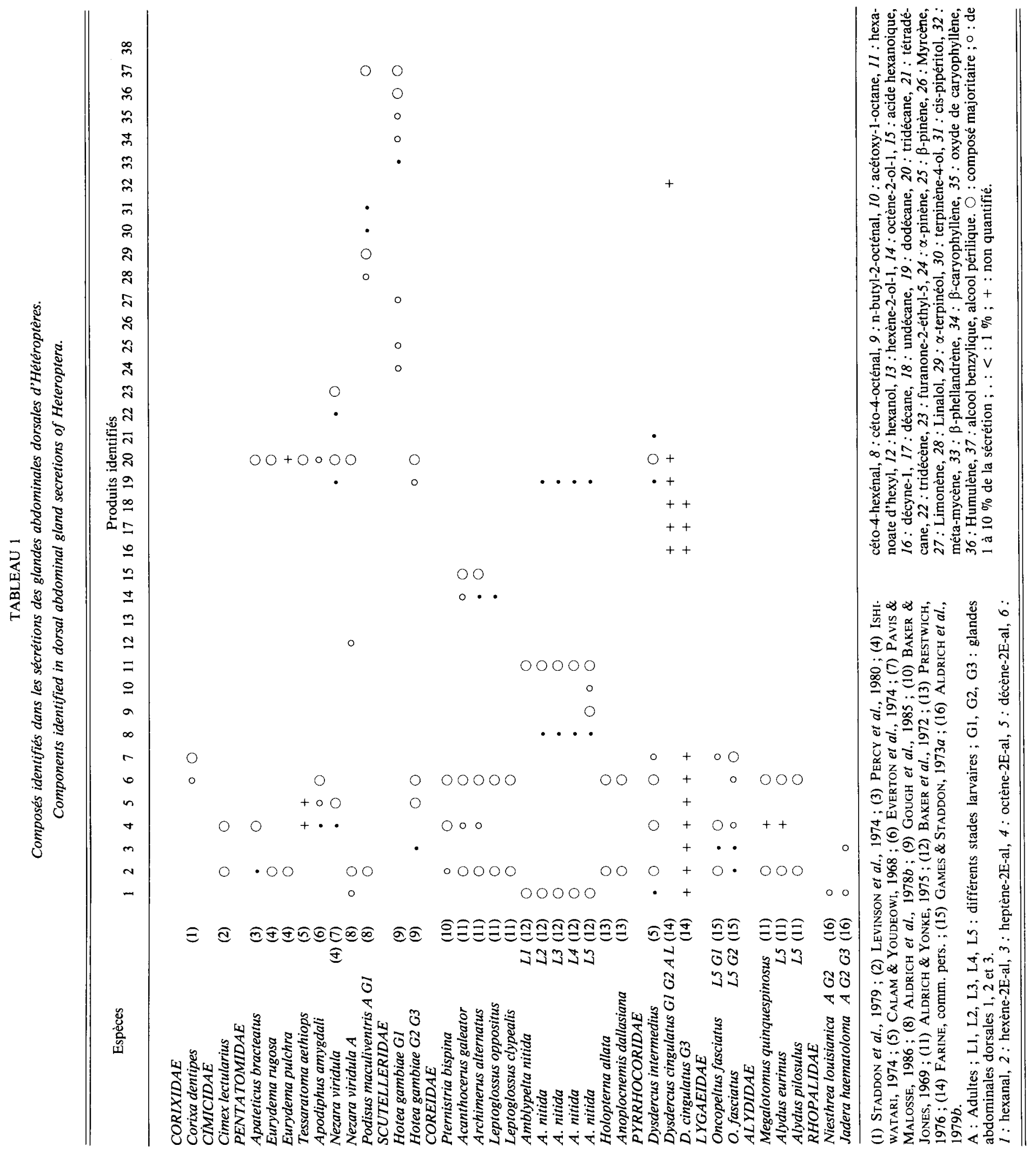


tenu des 3 réservoirs des glandes dorso-abdominales d'Hotea gambiae West. (Scutelleridae). Le réservoir antérieur contient un mélange de 8 terpènes alors que les 2 glandes postérieures produisent des aldéhydes et des hydrocarbures saturés.

Il apparaît donc que dans certains cas il existe une hétérogénéité, aussi bien du fonctionnement que de la nature des sécrétions produites par les différentes glandes dorso-abdominales de jeunes Hétéroptères, ceci suggérant des rôles différents.

Chez Dysdercus cingulatus Fabr., les sécrétions des glandes antérieures ont un rôle agrégatif ; celles de la glande postérieure assurent la défense contre les prédateurs (FARINE, comm. pers.).

Contrairement aux autres auteurs, BAKER et al. (1972) se sont intéressés à tous les stades larvaires chez un représentant des Coreidae : Amblypelta nitida Stal. Pour cette espèce, il apparaît que plus le stade est âgé et plus le nombre de composés contenus dans la glande augmente (un seul produit pour les $1^{\text {ers }}$ stades, 4 pour les $2^{\mathrm{e}}, 3^{\mathrm{e}}$ et $4^{\mathrm{e}}, 7$ produits pour les derniers stades). Cette étude semble donc indiquer qu'il existe un phénomène de maturation des glandes au cours du développement post-embryonnaire.

Bien que peu d'études en fassent état, les glandes abdominales dorsales des jeunes Hétéroptères sont loin de toujours régresser chez les adultes où il en subsiste presque toujours des traces, souvent même des restes encore fonctionnels (CARAYON, comm. pers.).

Chez les Pentatomoidea, la glande antérieure persiste et reste fonctionnelle. Dans le cas des phytophages et de quelques espèces prédatrices (ALDRICH et al., $1978 b$ ), ces glandes sont relativement petites et de même taille pour les 2 sexes ; mais chez quelques espèces d'Asopinae, dont Podisus maculiventris Say, la première glande des adultes mâles est extrêmement développpée.

Malheureusement, peu d'études physico-chimiques traitent de la nature des produits présents dans ces glandes. Chez Dysdercus intermedius (CALAM \& SCOTT, 1969) et $D$. cingulatus (FARINE, comm. pers.), les 3 glandes persistent et au moins 2 restent fonctionnelles.

ALDRICH et al. (1979b) ont étudié ces glandes chez les adultes de 2 espèces de Rhopalidae : Jadera haematoloma dont les glandes IV-V et V-VI persistent et Niesthrea louisianica Stal dont seule la glande médiane reste présente.

On trouve dans les sécrétions dorso-abdominales plusieurs types de composés :

- des composés aliphatiques saturés ou insaturés (de 6 à 10 atomes de carbone), portant le plus souvent une fonction aldéhyde mais parfois un alcool, un acide ou un ester. Le trans-hexène-2E-al est le composé que l'on trouve le plus fréquemment, aussi bien chez les Pentatomoidea que chez les Coreoidea ;

- des hydrocarbures saturés lourds, principalement le n-tridécane qui n'est présent que chez les Pentatomoidea (exception faite du genre Dysdercus) ;

- des monoterpènes et des sesquiterpènes. Pour l'instant, ils ont été identifiés chez peu d'espèces : jeunes d'Hotea gambiae West. (Scutelleridae) et adultes de Niesthrea louisianica Stal et Jadera haematoloma (Rhopalidae) ainsi que mâles de Podisus maculiventris Say (Pentatomidae).
Il est difficile de savoir si le contenu de ces glandes reste le même chez les adultes chez qui elles persistent car très rares sont les travaux qui mentionnent les 2 . Cependant, chez $D$. cingulatus, les adultes conservent des glandes moyennes et postérieures fonctionnelles. Elles contiennent les mêmes composés que celles des larves mais en quantités beaucoup plus importantes chez les femelles. On peut avancer l'hypothèse d'une phéromone sexuelle attractive pour les mâles.

\section{B. Les adultes}

\section{Position des glandes}

En sus des glandes abdominales dorsales qui peuvent persister à l'état adulte chez certaines espèces, on observe un appareil glandulaire plus complexe, le plus souvent situé ventralement sur le métathorax.

Certaines espèces sont dépourvues de cet appareil : c'est le cas des membres de la sous-famille des Belostomatinae (STADDON, 1971) ainsi que d'autres punaises aquatiques et de quelques Miridae.

\section{Structure (fig. 2)}

CARAYON (1971) a décrit plusieurs types d'appareils odorants métathoraciques. Le $1^{\mathrm{er}}$, propre à certains Reduvoidea est situé au niveau de la jonction thoraco-

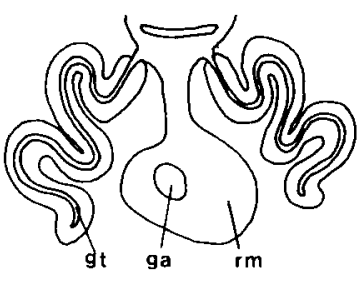

(a) Corixa dentipes (Corixidae)

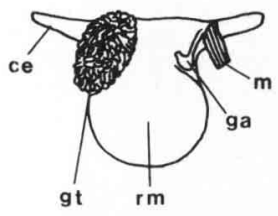

(c) Amorbus alternatus

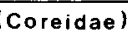

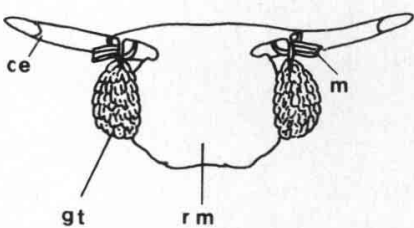

(b) Nezara viridula (Pentatomidae)

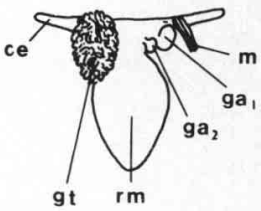

(c) Hyocephalus sp. (Hyocephalidae)

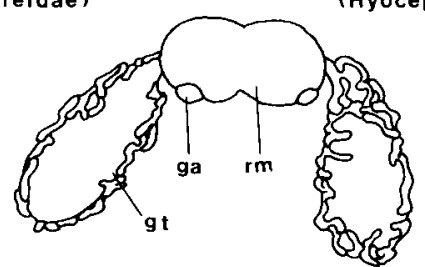

(d) Oncopeltus fasciatus

(Lygaeidae)

Figure 2

Schémas illustrant différents types de complexes métathoraciques chez quelques familles d'Hétéroptères. ce: canaux efférents; $m$ : muscles commandant l'ouverture des canaux efférents; $\mathrm{rm}$ : réservoir médian; ga: glande accessoire; gt: glande tubulaire. D'après STADDON et al. 1979 (a), GILBY \& WATHERHOUSE, $1967(b)$, WATERHOUSE \& GILBY, 1964 (c) et GAMES \& STADDON, 1973b (d).

Diagrammatic sketch of the scent gland complex of some Heteropteran families. ce: discharge canal, $m$ : opener muscles of the discharge canal ; rm : median reservoir ; ga : accessory glands ; gt : tubular glands. From STADDON et al. 1979 (a), GILBY \& WATERHOUSE, 1967 (b), WATHERHOUSE \& GILBY, 1964 (c) and GAMES \& STADDON, $1973 \mathrm{~b}(d)$. 
abdominale ventrale. Les 2 autres ont leur débouché au niveau du métathorax ventral, une distinction étant faite quant à la disposition des orifices de sortie des sécrétions : orifice impair et médian ou orifices extrêmement proches dans le cas des Hydrocorises, Amphibicorises et de quelques familles de Géocorises. Ce type est sans doute le plus primitif et dérive d'une simple invagination de la membrane articulaire thoracoabdominale, pourvue de cellules glandulaires uniformément réparties dans la paroi de cette invagination faisant office de réservoir.

Le type le plus évolué, propre à la plupart des Géocorises dérive du précédent par une bipartition de l'orifice et par une migration des 2 orifices en résultant, le réservoir restant impair quant à lui.

On observe évidemment des variations du complexe thoracique suivant les familles. D'une façon générale, il existe un réservoir impair médian possédant ses propres cellules glandulaires incluses dans l'épithélium du réservoir. Associées à ce réservoir, se trouvent une ou plusieurs paires de glandes de tailles et de formes diverses, souvent appelées glandes tubulaires ou accessoires.

Chez d'assez nombreux Lygaeidae, dont $O$. fasciatus, les glandes tubulaires possèdent leurs propres réservoirs dont le contenu sera déversé à son tour dans le réservoir médian (GAMES \& STADDON, 1973b).

Chez $D$. intermedius, le même schéma est respecté (EVERTON et al., 1979).

WATERHOUSE \& GILBY (1964) décrivent chez les Hyocephalidae 2 paires de glandes accessoires en plus des glandes tubulaires.

Le plus souvent, les parois du réservoir sont de couleur jaune ou orange ; elles contiendraient un ou plusieurs pigments à fonction métabolique. WATERHOUSE \& GiLBY (1964) suggèrent un rôle dans la détoxification des composés passant du réservoir vers l'hémolymphe.

\section{Fonctionnement}

La sortie des sécrétions est contrôlée par un système de verrouillage des canaux efférents ; la contraction des muscles insérés sur les canaux permet l'écoulement du liquide.

REMOLD $(1962,1963)$ décrit plusieurs façons d'émettre les substances odorantes. Chez la plupart des Pentatomidae, Anthocoridae, Coreidae, l'émission est unilatérale suivant le côté d'où vient l'agresseur et elle peut être assez large. Les Plataspidae du genre Coptosoma sont capables de viser assez précisément la source de perturbation. Certaines punaises (Anasa tristis De G., Tessaratoma papillosa Thunb. (Pentatomidae)) peuvent projeter le liquide à une distance allant jusqu'à $25 \mathrm{~cm}$ (WATERHOUSE et al., 1961).

Quelques Miridae quant à eux procèdent de façon particulière en humectant leurs tarses avec la sécrétion ; ils en frottent ensuite la source de perturbation. Une fois émise, la sécrétion se trouve au niveau des plaques ventrales qui peuvent prendre des formes diverses suivant les espèces ; ces plaques sont pourvues de structures cuticulaires en forme de champignons. Le rôle très souvent attribué à ces structures est l'augmentation de la surface d'évaporation (FILSHIE \& WATERHOUSE, 1969). Mais elles sont tellement petites qu'un très faible volume de liquide pourrait y rester ; elles ont plutôt un rôle hydrofuge et restent pratiquement sèches : lorsque la sécrétion arrive à leur niveau, elle se trouve repoussée sur une zone voisine du tégument (CARAYON, 1971). Ces microsculptures assurent donc une protection de l'insecte émetteur car grâce à elles, la sécrétion est chassée de la zone des stigmates thoraciques (CARAYON, 1971). On retrouve d'ailleurs au sein de l'ordre des Thysanoptères des structures analogues autour des stigmates abdominaux, permettant ainsi une protection contre la pénétration de l'eau dans le système trachéen.

\section{Sécrétions (tabl. 2)}

Chez la plupart des Pentatomoidea, le contenu du complexe glandulaire métathoracique est constitué de 2 phases non miscibles : une phase incolore contenant des hydrocarbures saturés (principalement le ntridécane) et une phase jaune où l'on trouve le plus souvent des aldéhydes et des esters.

La couleur jaune semble due à la présence de composés di-carbonylés (céto-4-hexénal, céto-4-octénal) dans la sécrétion. En effet, chez les Coreoidea, elle n'est constituée que d'une phase qui renferme aldéhydes et esters mais qui, elle, est incolore ; or, on ne trouve pas de composés dicarbonylés chez les Coreoidea sauf chez $O$. fasciatus (Lygaeidae) dont la sécrétion est justement jaune et constituée de 2 phases (GAMES \& STADDON, $1973 b$; EVERTON \& STADDON, 1979).

Nous constatons une différence entre Pentatomoidea et Coreoidea : les $1^{\text {ers }}$ synthétisent plutôt des composés insaturés et les $2^{\mathrm{e}}$ des composés saturés. Quelques exceptions existent et restent assez limitées sauf dans le cas d' $O$. fasciatus: cette espèce se détache de nouveau des Coreoidea par le fait qu'elle produit de l'hexénal, de l'octénal et de l'octadiénal en quantités importantes (un autre membre de la famille des Lygaeidae possède cette particularité : Oxycarenus hyalinipennis Costa (OLAGBEMIRO \& STADDON, 1983 ; KNIGHT et al., 1984).

Enfin, les sécrétions des Pentatomoidea contiennent très fréquemment des quantités importantes d'hydrocarbures, principalement le n-tridécane, dont sont dépourvus les représentants des Coreoidea.

Trois espèces de Pentatomidae font exception à cette règle : Dolycoris baccarum L., Eurygaster sp. et Palomena viridissima Poda qui produisent un aldéhyde saturé (hexanal), pas de composés dicarbonylés ni d'hydrocarbures saturés lourds comme le font les autres Pentatomoidea.

Quelques auteurs ont étudié séparément le contenu des différentes structures du complexe odorant métathoracique. GILBY \& WATERHOUSE (1967) montrent chez Nezara viridula L. que les glandes latérales contiennent des acétates (entre autres) alors que l'on trouve dans le réservoir médian les aldéhydes correspondants aux acétates cités plus haut (acétoxy-1-hexène, acétoxy-1-octène, acétoxy-1-décène).

Chez O. fasciatus, CARAYON (1948) décrit des glances tubulaires beaucoup plus grosses chez le mâle que chez la femelle. Chez le mâle, esters et aldéhydes correspondants existent dans les mêmes proportions mais chez la femelle, seuls les aldéhydes sont détectés. Pour cette même espèce, EVERTON \& STADDON (1979) confirment que les acétates sont localisés dans les glandes tubulaires et les aldéhydes dans le réservoir médian. 
TABLEAU 2

\section{HYDROCORISES}

\section{CORIXIDAE}

Sigara falleni

Corixa dentipes

(1)

NAUCORIDAE

(1) (2)

Ilyocoris cimicoides

(1)

BELOSTOMATIDAE

Lethocerus indicus

Notonecta glauca

GEOCORISES

CIMICIDAE

Cimex lectularius

MIRIDAE

(1) (4)

Leptopterna dolabrata

Harpocera thoracica

PENTATOMOIDEA

Apodiphus amygdali

Hyalomorpha mista

Menida scotti

Plautia stali

Biprorulus bibax

Musgraevia sulciventris

Podisus maculiventris

Nezara viridula

Poecilometis strigatus

Dolycoris baccarum

Eurygaster sp.

Palomena viridissima

Oebalus pugnax

Brachynema quadripustulata

Piezodorus teretipes

Tessaratoma aethiops

Aspongopus sp.

Delegorguella lautus

Caura rufiventris

Veterna patula

PLATASPIDAE

Coptosoma punctatissimum

Ceratocoris cephalicum

CYDNIDAE

Scaptocoris divergens

Macrocystus sp.

COREOIDEA

COREIDAE

Amblypelta nitida

Homoeocerus alternatus

Acanthocerus galeator

Archimerus alternatus

Leptoglossus oppositus

Merocoris distinctus

Amorbus rubiginosus

Amorbus alternatus

Amorbus rhombifer

Mictis profana

Mictis caja

Aulacosternum nigrorubrum

Pachylopura manca

Agriopocoris frogatti

Pternistria bispina

Acanthocephala femorata

Leptocoris apicalis

Anoplocnemis montandonii

Acanthocoris obscuricornis

Anoplocnemis dallasiana $\sigma$

Anoplocnemis dallasiana ९

Holopterna allata ơ mûr

$H$. allata $\bigcirc$ mure

$H$. allata or immature

PYRRHOCORIDAE

Dysdercus intermedius

Dysdercus cingulatus

Oxycarenus hyalinipennis 


\section{0}
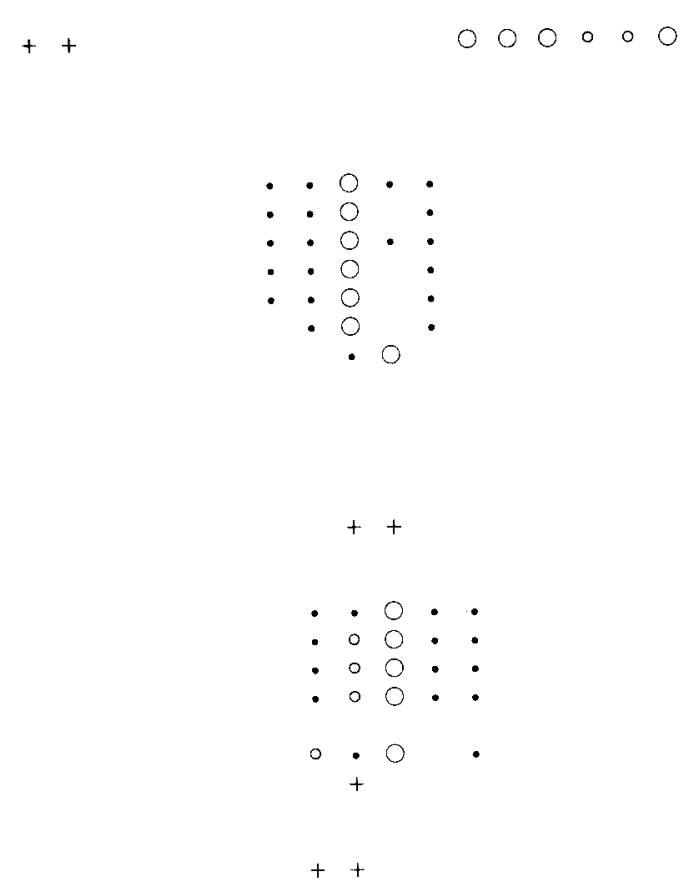

$\circ \mathrm{O}$

- 0

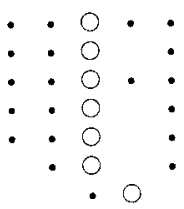

- 0 .

: 00 : 0

$+0$

$++$

0

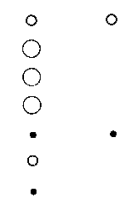


De même, EVERTON et al. (1979) mettent en évidence la présence d'acétoxy-1-hexène et acétoxy-1octène dans les glandes tubulaires et d'hexenal et d'octenal dans le réservoir médian de $D$. intermedius. Le tableau 2 montre également que chez de nombreuses espèces, aldéhydes et esters de la même famille sont présents simultanément.

Toutes ces constatations suggèrent le schéma suivant : les glandes tubulaires seraient responsables de la synthèse d'esters ; ces esters (surtout des acétates) transiteraient dans les réservoirs latéraux (dans le cas où ils existent) puis seraient déversés dans le réservoir médian où ils seraient en partie ou complètement transformés en aldéhydes grâce à des enzymes produits soit par l'épithélium même du réservoir soit par les glandes accessoires dont la paroi est munie.

Une autre famille de composés est parfois présente chez les Hétéroptères : les terpènes. On peut les trouver dans les glandes métathoraciques des adultes, comme c'est le cas pour Leptopterna dolabrata L. (Miridae), D. intermedius (Pyrrhocoridae), O. hyalinipennis (Lygaeidae), $N$. louisianica Stal (Rhopalidae). Chez cette dernière espèce, la sécrétion est totalement dépourvue de composés autres que terpéniques.

On trouve également des terpènes dans les glandes abdominales dorsales qui persistent chez les adultes de quelques espèces; c'est le cas pour $P$. maculiventris (Pentatomidae), $N$. louisianica et $J$. haematoloma (Rhopalidae).

Mais il est une toute autre famille de composés que nous n'avons pas mentionnée dans le tableau récapitulatif car leur structure exacte n'est pas connue, il s'agit des protéines. CARAYON (1971) a mis en évidence l'existence de mucopolysaccharides dans les sécrétions de très nombreuses espèces. Chez certaines familles telles que celle des Saldidae et des Eniocephalidae, la partie protéique peut atteindre les $3 / 4$ de la sécrétion; chez presque tous les Hétéroptères, on en trouve au moins des traces. Ces protéines sont produites au niveau des glandes annexes mais on n'en connaît ni la structure ni le rôle. Si ces produits ont été assez peu évoqués dans la littérature, c'est que les chimistes se sont surtout intéressés aux composés les plus faciles à analyser, en l'occurrence les composés carbonylés à chaîne relativement courte et les terpènes.

\section{Comparaison des sécrétions des larves et des adultes}

La composition des sécrétions des larves d'Hétéroptères a été moins étudiée que celle des adultes, cependant, quelques différences apparaissent. Là aussi, 2 grands groupes tendent à se détacher : Coreoidea et Pentatomoidea.

Chez ces derniers, il semble que la règle soit la similitude entre sécrétions des jeunes et des adultes, sauf pour Tessaratoma aethiops Dist. (Pentatomidae) où seul le décénal est commun aux deux (BAGGINI et al., 1966, in EVERTON et al., 1974).

Il semble que de telles différences existent plutôt au sein de la super-famille des Coreoidea: chez les 13 espèces pour lesquelles les 2 types de sécrétion ont été étudiés, toutes présentent des différences de compositions entre jeunes et adultes. L'amplitude de ces différences est variable : chez les adultes d'Amblypelta nitida Stal (Coreidae), on observe 6 composés communs avec les jeunes et quatre composés typiquement caractéristiques des adultes (BAKER et al., 1972).

Par contre, aucun des composés de la sécrétion des larves de Pternistria bispina Stal (Coreidae) n'est présent chez les adultes de cette même espèce (BAKER \& KeMBALl, 1967).

D'une façon générale chez les Coreoidea, les sécrétions des jeunes sont des mélanges moins complexes que chez les adultes et contiennent des composés plus insaturés que chez ces derniers ; or, un produit insaturé pénètre moins facilement dans la cuticule qu'un produit saturé. Pour BAKER \& JONES (1969), les différences de composition entre jeunes et adultes sont liées à des différences d'émission de la sécrétion : chez les jeunes, elle se fait par simple exsudation dorsale, les insectes se trouvant alors au contact des substances de défense ; cela ne les perturbe pas du fait qu'elles contiennent principalement des composés insaturés ayant un faible pouvoir de pénétration dans la cuticule. Par contre, les adultes projettent ventralement leurs sécrétions et ne se trouvent donc pas exposés aux composés saturés qui pénètreraient dans leur cuticule et auraient des effets toxiques. De plus, à l'inverse de celles des adultes, les sécrétions des jeunes Coreoidea contiennent fréquemment des composés di-carbonylés.

Note du tableau 2

(1) Calam \& Youdeowi, 1968 ; (2) Staddon et al., 1979 ; (3) Pattendon \& Staddon, 1968 ; (4) Levinson et al., 1974; (5) Collins \& DraKe, 1965 ; (6) HansSen, 1982 ; (7) Everton et al., 1974 ; (8) Kitamura et al., 1984 ; (9) MC Leod et al., 1975; (10) Aldrich et al., $1984 a$; (11) Aldrich et al., $1984 b$; (12) Gilby \& Waterhouse, 1967 ; (13) Waterhouse $e t$ al., 1961 ; (14) Prestwich, 1976 ; (15) Baker et al., 1972 ; (16) Aldrich \& Yonke, $1975 ;$ (17) Waterhouse \& Gilby, 1964 ; (18) BaKer \& Kemball, 1967; (19) EverTon et al., 1979; (20) Calam \& Scott, 1969 ; (21) Farine, comm. pers. ; (22) Games \& Staddon, $1973 b$; (23) Everton \& Staddon, 1979 ; (24) Olagbemiro \& StAdDON, $1983 ;$; (25) KNIGHT et al., $1984 ;$ (26) Aldrich et al., $1979 b$.

$1:$ éthanal, $2:$ butanal, $3:$ hexanal, $4:$ octanal, $5:$ propénal, $6:$ buténal, $7:$ hexène-2E-al, $8:$ hexène-2E-ol, $9:$ hepténal, $10:$ octénal, $11:$ décénal, $12:$ hexadiénal, $13:$ octadiénal, $14:$ décadiénal, $15:$ méthyl-2-propanal, $16:$ méthyl-2-butanal, $17:$ céto-4-hexénal, $18:$ céto-4octénal, $19:$ butyl-2-octénal, $20:$ acétoxy-1-hexane, $21:$ acétoxy-1-octane, $22:$ acétoxy-1-hexène, $23:$ acétoxy-1-octène, 24:acétoxy-1-décène, $25:$ acétoxy-1-hexadiène, $26:$ acétoxy-1-octadiène, $27:$ propanoate de diméthyl-propane, $28:$ propanoate de diméthyl-butane, $29:$ butanoate de butane $30:$ butanoate d'hexane, $31:$ butoxy-1-hexène-2E, $32:$ butanoate de diméthyl-butane, $33:$ butanoate de tridécane, $34:$ butanoate de tétradécane, $35:$ hexanoate de butane, $36:$ hexanoate d'hexane, $37:$ hexanoate de méthyl-butane, $38:$ butanol-1, $39:$ hexanol-1, $40:$ tridécanol, $41:$ décénol, $42:$ hexadiénol, $43:$ octadiénol, $44:$ céto-4-hexène-2E-al, $45:$ butanone- $2,46:$ hexanone, $47:$ nonanone, $48:$ acide acétique, $49:$ acide butanoïque, $50:$ acide hexanoïque, $51:$ acide méthyl-2-propanoïque, $52:$ acide méthyl-2-butanoïque, $53:$ parahydroxybenzaldéhyde, $54:$ parahydroxybenzoate de méthyl, $55:$ undécane, $56:$ dodécane, $57:$ tridécane, $58:$ tétradécane, $59:$ pentadécane, $60:$ géraniol, $61:$ géranial, $62:$ acétate de géraniol, $63:$ nérol, $64:$ néral, $65:$ acétate de nérol, $66: \alpha$-pinène, $67: \beta$-pinène, $68:$ myrcène, $69:$ limonène, $70:$ thymol, $71:$ cinéole, $72:$ linalol, $73: \alpha$-terpinène, $74:$ terpinolène, $75:$ terpinénol-4, $76:$ pipéritone, $77: \alpha$-farnesène, $78:$ méta-cymène.

0 : composé majoritaire ; $0:$ de 1 à $10 \%$ de la sécrétion $;.:$ inférieur à $1 \%$; + : non quantifié. 


\section{Rôle des sécrétions abdominales et thoraciques}

\section{Adultes}

La fonction la plus souvent avancée et la mieux connue de ces sécrétions odorantes des punaises est la défense non spécifique contre les agresseurs.

Chez certaines punaises aquatiques telles que Notonecta glauca L. (Notonectidae), Iliocoris cimicoides L. (Naucoridae), on trouve des composés aromatiques (phydroxybenzaldéhyde, p-hydroxybenzoate de méthyl) dont le rôle serait de les protéger contre certains microorganismes (PATTENDON \& STADDON, 1968) ; ces substances se comporteraient donc comme des antiseptiques. ROTH (1961) quant à lui attribue une action fongicide aux sécrétions des Scaptocoris.

Lorsqu'elles ont pour but la défense, ces substances agissent comme des irritants de contact contre un large spectre de prédateurs vertébrés ou invertébrés (EISNER, 1970 in ALDRICH \& YONKE, 1975). WATERHOUSE et al. (1961) ont observé plusieurs espèces de Pentatomidae et de Coreidae se défendre de cette façon contre des fourmis, des amphibiens, des souris et même des oiseaux. Pour ces derniers, il faut que les yeux soient atteints pour déclencher un effet répulsif. Une espèce africaine d'Aspongopus produit des sécrétions très irritantes pour les yeux de l'homme, quelques cas de cécité ont même été signalés. Pour certaines espèces de punaises, le contact avec l'agresseur n'est pas nécessaire pour déclencher l'émission : des mouvements ou des vibrations de ce dernier peuvent être suffisants.

Le composé le plus fréquemment identifié est l'hexène-2E-al. Il est intéressant de noter que cette molécule est produite par de nombreux végétaux et qu'elle exerce sur des insectes autres que les punaises des effets variés. Pour le ver à soie, elle agit comme phagostimulant.

Chez les Pentatomoidea, la sécrétion défensive est le plus souvent constituée d'aldéhydes insaturés ou saturés et de $n$-tridécane. Le tout forme un liquide lipophile qui se répand donc rapidement sur la cuticule de l'agresseur ; la surface d'action de la substance toxique est donc importante.

La cuticule des agresseurs est perméable aux sécrétions. REMOLD (1963) a montré que les aldéhydes seuls provoquent la paralysie du prédateur mais que la présence de n-tridécane (non toxique par lui-même) augmente la vitesse de pénétration des aldéhydes dans la cuticule.

Si le poison pénètre dans les trachées par un stigmate, l'effet est beaucoup plus rapide et violent : la mort peut suivre.

Mais en plus du rôle offensif direct, il existe aussi une action répulsive, en particulier vis-à-vis des fourmis. Dans ce cas, il n'y a pas contact direct mais agression olfactive contre la source de perturbation. Le tridécane joue alors le rôle de solvant pour les aldéhydes et évite une évaporation trop rapide de la sécrétion.

KITAMURA et al. (1984), lorsqu'ils ont mené l'étude physico-chimique des sécrétions ont fait une distinction entre les produits recueillis après collecte d'effluves (donc les plus volatils) et après rinçage de l'insecte avec un solvant. Ils en ont déduit que chez les Pentatomoidea, le n-tridécane et le décénal jouent le rôle de solvants car on ne les retrouve qu'en très petites quan- tités dans les collectes d'effluves. La cétone serait dans ce cas la substance toxique.

Pour les Coreoidea, le schéma est différent : l'hexanoate d'hexane serait le solvant et l'hexanal la substance active.

Dans le cas où elles assurent défense ou protection, les sécrétions que nous avons évoquées se conduisent donc comme des allomones, c'est-à-dire des substances émises par une espèce à destination d'une autre espèce au bénéfice de l'espèce émettrice.

CALAM \& YOUDEOWI (1968) ont montré que chez $D$. intermedius, l'hexénal, le buténal et la butanone provoquaient chez les adultes une excitation se traduisant par des mouvements, un arrêt du nettoyage des antennes, parfois l'ouverture des ailes et la défécation. Esters et acides seuls ne provoquent aucun de ces signes d'irritation; ils joueraient donc le rôle de solvants et d'agents mouillants pour les substances actives qui se comporteraient donc comme des phéromones d'alarme.

ALDRICH \& YONKE (1975) ont montré qu'au sein d'un même genre (Leptoglossus, Alydus...) les sécrétions étaient assez uniformes et qu'il n'existait pas de spécificité au niveau de l'espèce. Mais ils pensent que la sécrétion, associée à d'autres odeurs telles que celles produites par les glandes paragénitales décrites par BOHNAG \& WIG en 1953 constituerait une odeur propre à l'espèce importante dans les phénomènes de reconnaissance interindividuelle et se comporteraient comme une phéromone.

Chez les Belostomatidae, Lethocerinae, le dimorphisme sexuel des glandes métathoraciques ventrales est extrêmement accusé. L'appareil glandulaire des femelles est considérablement réduit par rapport à celui des mâles : chez ces derniers, les glandes se dilatent énormément à maturité sexuelle. BUTENAND \& TAM (1957) ont analysé le contenu de ces glandes : on y trouve de l'acétoxy-1-hexène-2E-al et du butoxy-1hexène-2E qui ont une action toxique ; mais lorsque les mâles sont sexuellement actifs, leurs glandes ne produisent plus que l'alcool correspondant aux 2 composés cités plus haut et cet alcool n'est pas toxique. Ces observations suggèrent un rôle sexuel de ces sécrétions mais l'expérimentation reste à faire.

D'autre part, on observe des différences entre les sécrétions des femelles et des mâles d'O. fasciatus. Chez ces derniers, il y a production d'acétates alors que les femelles en sont dépourvues, ce qui suggère un rôle sexuel de ces composés, qui reste encore à prouver. Le contenu du réservoir médian (aldéhydes et éventuellement acétates) englobe une gouttelette d'eau, produite par les glandes accessoires. Le rôle de cette eau serait multiple (EVERTON \& STADDON, 1979). D'une part, l'eau augmente la tension de surface de la sécrétion, ce qui permet la formation d'une gouttelette qui peut être manipulée par les tarses de l'insecte. D'autre part, sa présence augmente le volume du liquide émis : en effet, étant de type diastomien, le complexe métathoracique d'O. fasciatus produit des volumes très faibles de sécrétion. Une autre fonction est envisagée pour cette eau : elle participerait à la formation extra-cellulaire des aldéhydes du réservoir médian en décrochant les esters des parois des glandes accessoires.

Chez Harpocera thoracica Fal. (Miridae), on 
observe également un dimorphisme sexuel des sécrétions : les femelles produisent des acétates mais pas les mâles ; le rôle sexuel éventuel reste à vérifier (HANSSEN \& JACOB, 1982).

Le rôle des terpènes chez les punaises est encore mal connu. Les seules certitudes dans l'état actuel de nos connaissances sont que le linalol stimule la prise de nourriture chez $D$. intermedius : cela se traduit par des mouvements du rostre si le linalol est présenté seul aux insectes et à une prise alimentaire plus rapide et plus importante s'il est associé à sa nourriture (EVERTON $e t$ al., 1979).

De plus, GHandi (in Olagbemiro \& Staddon, 1983) a mis en évidence un effet répulsif de l' $\alpha$-pinène sur $O$. hyalinipennis.

Ces données sont assez fragmentaires mais on peut s'intéresser à ce qui se passe chez d'autres insectes :

$-\alpha$ et $\beta$-pinène sont des substances de défense des résineux contre les larves de Neodiprion sertifer Geoffroy (EISNER et al., 1971) ;

- le limonène est une phéromone d'alarme chez les termites (MOORE, 1968). Chez ces derniers, les terpènes sont des substances de défense ;

- certains sesquiterpènes jouent le rôle de phéromones d'alarme chez les pucerons ;

- géraniol, nérol et linalol sont des phéromones d'agrégation chez les abeilles solitaires du genre Colletes;

- chez certaines plantes, l' $\alpha$-farnesène est un attractif pour des espèces d'insectes phytophages et un répulsif pour d'autres ;

- le géraniol est une phéromone d'alarme chez les fourmis (BLUM et al., 1968).

On se trouve donc pour l'instant réduit à faire des hypothèses quant au rôle des terpènes chez les punaises.

KNIGHT et al. (1984) pensent que l' $\alpha$-farnesène rencontré chez $O$. hyalinipennis aurait un rôle défensif contre les fourmis et les termites. Les adultes de cette espèce se frottent le corps avec leurs tarses postérieurs et on retrouve l' $\alpha$-farnesène dans la région des segments pré et paragénitaux ce qui suggère un phénomène d'auto-marquage avec la sécrétion thoracique ; ceci aurait pour but de signaler la maturité sexuelle car les terpènes n'apparaissent que lorsque cette maturité est atteinte. Le rôle de cette molécule pourrait être également d'attirer d'autres individus sur un lieu favorable d'un point de vue trophique.

Chez D. intermedius, EVERTON et al. (1979) tentent d'expliquer le rôle de linalol de différentes façons : étant un isomère proche du géraniol, la phéromone d'alarme produite par les glandes mandibulaires des fourmis (BLUM et al., 1968), il pourrait s'agir d'un mimétique de cette phéromone ; ainsi, $D$. intermedius se trouverait protégé des fourmis par la fuite de cellesci. Ces auteurs pensent même que la stimulation à la prise de nourriture provoquée par le linalol aurait un effet sélectif car il provoque le cannibalisme des jeunes vis-à-vis des vieux, mois aptes à se défendre et participerait ainsi au maintien de populations saines. Une objection peut être faite à cette explication car dans la nature, les insectes ne se trouvent pas en concentrations telles qu'un phénomène de cannibalisme puisse réguler les niveaux de population.

On trouve également des terpènes chez $P$. maculi- ventris, mais au niveau des glandes abdominales dorsales qui persistent chez les adultes de ce Pentatomidae. Les glandes antérieures des mâles sont très développées et contiennent un mélange terpénique assez complexe (terpinéol, linalol, terpinène-4-ol, pipéritol). $\mathrm{Ce}$ mélange est attractif à longue distance pour les mâles, les femelles et certains parasites. Il s'agit donc à la fois d'une kairomone et d'une phéromone d'agrégation (ALDRICH et al., 1984a). Les glandes des femelles produisent des composés absents chez les mâles (benzaldéhyde, acide hexanoïque, octénal, nonanal). ALDRICH et al. (1984b) suggèrent qu'il s'agisse d'un attractif à courte distance pour les mâles.

\section{Jeunes}

Les jeunes Hétéroptères, surtout lors de leurs premiers stades larvaires, forment assez souvent des agrégats; l'avantage de ce type de formation est que si un ou plusieurs jeunes ont trouvé une situation propice, tant au niveau de la source de nourriture que d'une protection par un élément naturel, les autres en profitent. Si un danger se présente, ou un dérangement quelconque, les groupes se dissocient mais se reforment au bout d'un temps variable. Certains travaux montrent que les sécrétions dorso-abdominales des jeunes jouent un rôle dans la formation de ces groupes aussi bien que dans leur rupture.

ISHIWATARI $(1974,1976)$ a montré chez 3 espèces de Pentatomidae (Eurydema pulchra West., Eurydema ruguosa Motsch., Nezara viridula) que l'hexène-2E-al se comportait à la fois comme une phéromone d'agrégation et comme une phéromone d'alarme. Ceci peut être expliqué par des différences quantitatives d'émission odorante; lorsqu'un individu, au sein d'un groupe est perturbé, il émet rapidement une quantité importante d'hexène-2E-al, ce qui provoque l'alarme et la dispersion des autres membres du groupe. Relâchée progressivement et en faibles quantités, la sécrétion agit alors comme une phéromone d'agrégation par son action attractive et d'arrestation.

Chez les larves de $D$. intermedius, les 2 premières glandes produisent du n-tridécane et la $3^{\mathrm{e}}$ de l'hexène2E-al. Lorsqu'un individu est agressé, il décharge le contenu de la $3^{\mathrm{e}}$ glande (rarement celui des 2 premières) qui s'associe alors à une gouttelette de sécrétion anale. Ceci lui permet de se défendre contre l'agresseur mais provoque également la fuite des autres membres de l'agrégat par son effet répulsif ; il s'agit donc d'une phéromone d'alarme (YoudEowi \& CALAM, 1969).

Pour cette espèce, l'hexène-2E-al ou le décène-2E-al seuls provoquent l'éclatement des groupes mais pas les autres composés pris isolément. De plus, on observe souvent les jeunes se passer les tarses sur les débouchés des glandes puis se les frotter et se nettoyer les antennes ; on peut penser qu'en se comportant ainsi, ils se répandent une phéromone d'agrégation sur le corps. En outre, si l'on obstrue l'ouverture des glandes, l'agrégation est fortement inhibée.

L'existence des terpènes dans les sécrétions des jeunes n'est connue que chez Dysdercus cingulatus (Phyrrhocoridae) et Hotea gambiae (Scutelleridae); il est intéressant de noter que ces terpènes sont très souvent présents chez des punaises associées à des Malvacées; des études physico-chimiques de ces plantes ont 
montré qu'on n'y trouvait pas les terpènes fabriqués par les insectes : ils les synthétiseraient eux-mêmes.

Ces isoprénoïdes ont certainement un rôle défensif. HONDA (1981) a montré que l'oxyde de caryophyllène (produit par $H$. gambiae) avait un effet répulsif sur les fourmis. Chez certaines Légumineuses tropicales, cette même substance agit comme antifongique (HUBELL et al., 1983).

\section{AUTRES FORMATIONS GLANDULAIRES}

\section{A. Cas des Anthocoridae}

Les mâles de certains genres appartenant à cette famille (Scolopa, Scolopella, Scoloposcelis) possèdent dans l'abdomen au niveau des segments III, IV et V des sacs glandulaires relativement volumineux dont la formation a lieu au dernier stade larvaire (CARAYON, 1954). Ils contiennent un liquide huileux dont la fonction n'est pas connue. On peut seulement avancer l'idée d'une fonction corrélée à la reproduction puisque seuls les mâles possèdent cet appareil glandulaire.

\section{B. Cas des Lygaeoidea, Pyrrhocoroidea, Coreoidea}

Au sein de ces groupes, d'anciens auteurs (MAYER, 1873 ; LUDWIG, 1926 ; GUPTA, 1951 ; BOHNAG \& WIG, 1953 ; STYS, 1959 in THOUVENIN, 1965) ont mentionné chez certaines espèces des glandes ectodermiques présentant souvent un dimorphisme sexuel, au niveau des derniers segments abdominaux ventraux. ThOUVENIN (1965) a établi que ces glandes étaient générales aux Pentatomorphes (exceptés les Pentatomoidea) et leur a attribué le nom d'« uradénies » (fig. 3).

Chez les Lygaeoidea, ces glandes sont peu répandues mais lorsqu'elles existent, elles sont différentes d'un sexe à l'autre.

Chez les Pyrrhocoroidea, ces uradénies n'existent que chez les femelles, les mâles en étant totalement dépourvus. Rien n'est connu de la nature ni du rôle des sécrétions de ces glandes.

Enfin, le cas des Coreoidea semble un peu mieux connu. Les femelles en possèdent rarement, sauf en ce
(A)

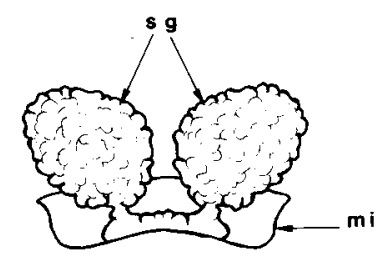

(B)

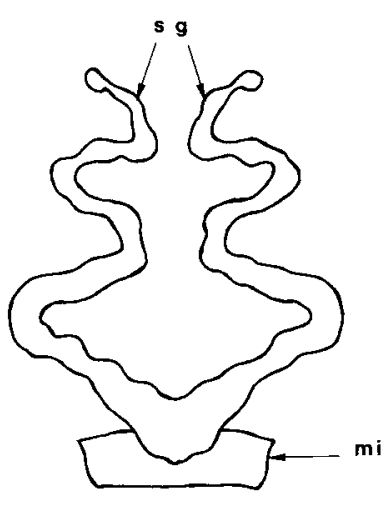

Figure 3

Schéma de deux types d'" uradénies " montrant les différences pouvant exister dans la forme des sacs glandulaires et dans les débouchés des glandes qui peuvent être pairs ( $A$ : mâle de Dicranocephalus agilis $H a h n$ (Stenocephalidae)) ou impairs ( $B$ : mâle de Dalader acuticosta $A . \& S$. (Coreidae)). sg : sac glandulaire; mi : membrane intersegmentaire VII-VIII. D'après THOUVENIN, 1965.

Two types of uradenia showing the variation of size and shape. sg: gland sac ; mi : VII-VIII intersegmental membrane. (A : male of Dicranocephalus agilis Hahn (Stenocephalidae), $B:$ male of Dalader acuticosta (A.\& S.) (Coreidae)). From THOUVENIN, 1965.

qui concerne quelques Coreidae. Par contre, on trouve des uradénies chez les mâles de toutes les familles de Coreoidea.

Lorsque les mâles sont sexuellement actifs, la glande est distendue car elle renferme une sécrétion qui a été analysée chez quelques espèces (tabl. 3). On trouve le plus souvent des alcools aromatiques dans ces sécrétions, sauf dans le cas de Pachylis laticornis F. dont les glandes contiennent des esters (ALDRICH et al., 1982).

Ces substances semblent liées à l'activité sexuelle, car chez les mâles fraîchement émergés, les glandes

TABLEAU 3

Composés identifiés dans les « uradénies " de mâles de Coreidae.

Components of the secretions from the ventral abdominal glands uradenia of adult male coreids.

\begin{tabular}{|c|c|c|c|c|c|c|c|c|c|c|c|c|c|c|c|c|}
\hline \multirow{2}{*}{ Espèces } & & \multicolumn{15}{|c|}{ Produits identifiés } \\
\hline & & 1 & 2 & 3 & 4 & 5 & 6 & 7 & 8 & 9 & 10 & 11 & 12 & 13 & 14 & 15 \\
\hline Pachylis laticornis & (1) & & & & & & & & & & & & 0 & 0 & $\circ$ & $\circ$ \\
\hline Leptoglossus phyllopus & (2) & 0 & & 0 & & $\circ$ & & & & $\circ$ & $\circ$ & & & & & \\
\hline Leptoglossus corculus & (2) & 0 & 0 & & 0 & & 0 & o & & $\circ$ & & & & & & \\
\hline Leptoglossus fulvicornis & (2) & 0 & & 0 & & & & & & & & & & & & \\
\hline Leptoglossus oppositus & (2) & 0 & & & & & & & & & & & & & & \\
\hline Leptoglossus clypealis & (2) & 0 & & 0 & & & & & & & & & & & & \\
\hline Leptoglossus gonagra & (2) & & & & & & & & 0 & & & & & & & \\
\hline Eutochta galeator & (2) & 0 & & & & & & & 0 & & & & & & & \\
\hline
\end{tabular}

0 : composé majoritaire, $\circ:$ de 1 à $10 \%$ de la sécrétion, . : inférieur à $1 \%$.

1: Alcool benzylique, $2:$ n-octanol, $3:$ Guaiacol, $4:$ Parahydroxybenzaldéhyde, $5:$ Parahydroxybenzoate de méthyl, $6:$ Vanilline, $7:$ Alcool de vanilline, $8: 2$-phényl-éthanol, $9:$ Alcool cynamique, $10:$ Syringaldéhyde, $11:$ Acétosyringone, $12:$ Tiglate d'hexène, $13:$ Hexanoate d'hexène, $14:$ Tiglate d'octène, $15:$ Benzoate d'hexène.

(1) AldRICH et al., 1982 ; (2) AldRICH et al., 1979a. 
peuvent être vides comme chez Leptoglossus phyllopus L. (ALDRICH et al., 1976) ou ne pas contenir la totalité des composés présents chez l'adulte mûr (cas de $P$. laticornis cité plus haut).

Mais ces composés ne jouent pas un rôle sexuel à courte distance car les mâles à qui on a supprimé les glandes s'accouplent très bien lorsqu'ils sont mis à proximité des femelles.

ALDRICH et al. (1979a) ont étudié ces sécrétions chez 6 espèces du genre Leptoglossus et chez Eutochtha galeator F. Ils sont arrivés à la conclusion qu'elles étaient spécifiques du sexe et aussi de l'espèce, qualitativement et quantitativement.

On peut donc penser que ces glandes abdominales de mâles de Coreidae produisent des sécrétions qui attirent les femelles de leur propre espèce à longue distance (une position d'appel, avec le segment abdominal VII en extension est décrite chez $L$. phyllopus). Ce rapprochement des sexes favorise bien sûr la reproduction mais il permet aussi de limiter les dépenses énergétiques des femelles qui arrivent ainsi directement sur des lieux offrant des possibilités trophiques. Cette phéromone sexuelle constituerait donc aussi une adaptation à la colonisation d'habitats favorables.

\section{Cas des Pentatomoidea}

La plupart des mâles appartenant à cette superfamille possèdent des glandes unicellulaires dans leur tégument abdominal ventral ; les femelles en sont partiellement ou totalement dépourvues (CARAYON, 1981). Ces glandes tégumentaires particulières sont toutes du même type fondamental mais on observe bien sûr des variations quant à leur répartition, leur densité, la forme de leur canalicule excréteur, de leur orifice de sortie et des différenciations cuticulaires qui peuvent y être associées.

L'activité de ces glandes est fortement corrélée à l'activité sexuelle. Chez $N$. viridula (Pentatomidae), les mâles sexuellement immatures ou en quiescence voient leurs formations glandulaires régresser ; elles ressemblent alors à celles des femelles. Lorsque l'activité sexuelle reprend, les cellules glandulaires redeviennent volumineuses et fonctionnelles. Chez cette espèce, MitCHELl \& MAU (1971) ont montré que les mâles sexuellement mûrs étaient attractifs pour les femelles, au moins à faible distance (les expériences au champ n'ont rien donné). BRENNAN et al. (1977) confirment l'attraction à courte distance et apportent quelques précisions : les femelles ne sont attirées qu'après une semaine de vie imaginale, les mâles sont attractifs au bout de 4 jours et ils ne perdent pas leur attractivité après s'être accouplés.

PAVIS \& MALOSSE (1976) ont montré que cette attraction à courte distance est au moins en partie olfactive : des extraits de mâles sexuellement mûrs sont attractifs pour des femelles, également arrivées à maturité.

Enfin, HARRIS \& TODD (1980) mettent en évidence par des expériences " grandeur nature " au champ une attraction à longue distance par les mâles mûrs des femelles mais aussi des mâles, des derniers stades larvaires et même d'un parasite.

Deux phénomènes semblent donc se superposer : une attraction à courte distance où l'olfaction et l'audition jouent certainement un rôle (la stridulation a été décrite fort précisément chez cette punaise par HARRIS et al. (1982)) et une attraction à longue distance à effet agrégatif : on aurait donc affaire à une phéromone sexuelle d'une part et à une phéromone d'agrégation doublée d'une kairomone d'autre part.

On soupçonne les glandes unicellulaires abdominales des mâles d'être responsables de la production de cette ou de ces phéromones.

Chez 3 espèces de Pentatomidae Asopinae (Perillus bioculatus F., Stiretrus anchorago F. et Oplomus severus Bred.), ALDRICH et al. (1986) décrivent également des glandes épidermiques ventrales, absentes chez les femelles, qui produisent principalement de l'isovalérate de 6,10,13-tétradécane et du triméthyl-6,10,13tétradécanol. La fonction de ces sécrétions mâles n'est pas connue.

Chez un autre Pentatomoidea, Eurygaster integriceps Put. (Scutelleridae), les mâles produisent sans doute au niveau de ces glandes abdominales un mélange de vanilline et d'acrylate d'éthyl qui est un stimulant sexuel et un attractif à courte distance pour les femelles (VRKOC et al., 1977). Dans ce cas, vanilline ou acrylate d'éthyl seuls provoquent des réponses de la part des femelles mais ces réponses sont plus marquées si les deux composés sont en mélange : ils agissent donc en synergie.

Chez Plautia stali Scott (Pentatomidae), les mâles adultes attirent mâles et femelles (MORIYA \& SHIGA, 1984) ; ils produisent donc une phéromone d'agrégation dont la structure n'est pas connue mais dont on peut penser qu'elle est sécrétée au niveau du tégument abdominal ventral des mâles, richement pourvu de glandes tégumentaires unicellulaires. Il est à noter que pour cette espèce, l'accouplement ne suit pas l'attraction comme chez $E$. integriceps.

Ces glandes tégumentaires sont parfois associées à des poils dressés qui ont pour but de retenir les masses friables et solides de sécrétions qui se détacheront à l'occasion de contacts avec la femelle ; c'est le cas chez quelques Pentatomidae Asopinae (Stiretrus, Discocera, Dorycoris, Cazira).

Le tégument peut présenter des différenciations plus ou moins marquées au niveau de ces glandes. Dans les genres Tectocoris, Psacata, Odontoscelis, Irochrotus (Scutelleridae), ces différenciations prennent la forme de longs processus ciliaires creux semblables aux androconies des Lépidoptères, qui sont emplis de sécrétions solides (CARAYON, 1984). Le contenu de ces androconies est libéré lors de leur rupture, ces formations étant dépourvues de canalicules de sortie ; la libération des sécrétions survient lors des contacts avec la femelle. On a sans doute affaire à une phéromone aphrodisiaque qui agirait par simple contact.

On trouve aussi des androconies dans les genres Solenosthedium, Coleothicus et Hotea (Scutelleridae) mais elles sont plus petites et moins complexes que celles décrites ci-dessus.

\section{Cas de Dysdercus cingulatus Fabr.}

Chez ce Pyrrhocoridae, LAWRENCE \& STADDON (1975) ont décrit certaines particularités du système glandulaire épidermique des adultes, situé sur le tégu- 
ment abdominal ventral. Ils ont observé 3 types de glandes, dont certaines présentent un dimorphisme sexuel.

Les premières, nommées "glandes simples ", sont en relation avec les phénomènes de mue et sont présentes chez les 2 sexes.

En outre, il existe des «glandes florales » et des " glandes-alvéoles », uniquement chez les mâles. Réduites et vides juste après la mue imaginale, elles se distendent ensuite et s'emplissent de sécrétions lorsque les mâles atteignent la maturité sexuelle. FARINE (comm. pers.) a identifié 2 terpènes ( $\beta$-caryophyllène et $\alpha$-humulène) au niveau de cet épiderme glandulaire, qui semblent avoir une action aphrodisiaque sur les femelles.

\section{E. Autres cas}

D'autres membres de l'ordre des Hétéroptères produisent des phéromones mais on ne connaît ni leur nature chimique ni la localisation des structures qui les produisent.

\section{Phéromones mâles}

LENER (1967, 1969) in BALDWIN et al. (1971) met en évidence la production d'un attractif sexuel par les mâles d' $O$. fasciatus.

Chez Rhodnius prolixus Stal, il se passe quelque chose de plus complexe : en plus d'une phéromone produite par les mâles, attractive pour les mâles, les femelles et les jeunes, les insectes in copula sont attractifs et excitants pour les mâles uniquement. Si le mâle et la femelle sont séparés, l'effet disparaît. Pour cette espèce, la communication serait donc assurée par différents types de médiateurs : phéromone d'agrégation, phéromone sexuelle et aphrodisiaque.

\section{Phéromones femelles}

Deux espèces appartenant à la famille des Pyrrhocoridae utilisent pour le rapprochement des sexes une phéromone produite par les femelles. Il s'agit de D. cingulatus (OSMANI \& NAIDU, 1967 in KING, 1973) et de Pyrrhocoris apterus L. (ZDAREK, 1970 in LAWRENCE \& STADDON, 1975).

Dans la famille des Miridae, il semble que la production de phéromones soit réservée aux femelles.

En 1968, SCALES démontrait que les femelles vierges de Lygus lineolaris Pal. de B. attiraient les mâles de cette espèce.

STRONG et al. (1970) ont mis en évidence le même phénomène chez Lygus hesperus Knight en précisant que l'attraction avait lieu le matin et pas avant 6 jours de vie imaginale ; cette attractivité cesse immédiatement après l'accouplement mais elle reprend quelques jours après. La production de phéromone s'arrête pendant la diapause.

Enfin, KING (1973) a décrit une position d'appel chez Distantiella theobroma Dist.. Chez cette espèce, les femelles exercent une attractivité sur les mâles, 3 à 4 jours après la mue imaginale et plutôt en fin d'aprèsmidi. Contrairement à l'exemple précédent, ce phénomène disparaît après l'accouplement.

Le cas des Miridae n'est pas sans rappeler celui de nombreux Lépidoptères nocturnes : les phéromones sont produites par les femelles au bout de quelques jours de vie imaginale, à une période bien précise du rythme nycthéméral, avec parfois une position d'appel particulière.

\section{CONCLUSION}

Les Hétéroptères sont très riches en glandes variées dont les plus étudiées sont les glandes métathoraciques des adultes, tant au niveau de leur structure que de la nature de leurs sécrétions. Elles sont de natures diverses et présentent en nombre et en proportions plus ou moins importants des composés appartenant à de nombreuses familles (aldéhydes, esters, acides, alcools, cétones, terpènes, composés aromatiques et protéines). Ces produits ont une valeur chémotaxonomique au niveau de la super-famille (Pentatomoidea et Coreoidea s'opposent nettement) et leur principale fonction est défensive.

Bien que moins étudiées, les glandes abdominales dorsales des jeunes stades sont extrêmement intéressantes car leur rôle est multiple. Deux types de sécrétions semblent ici intervenir : l'une de type allomone assure la défense et l'autre de type phéromone déclenche alarme ou agrégation.

Chez certaines espèces pour lesquelles ces glandes persistent à l'état adulte, il semble qu'elles puissent évoluer et parfois jouer un rôle dans la communication sexuelle en produisant des phéromones. A la fonction ancestrale défensive pourrait donc se superposer une fonction sexuelle apparue au cours de l'évolution.

En ce qui concerne les autres formations glandulaires, on rencontre dans presque toutes les familles d'Hétéroptères des glandes variées présentant un dimorphisme sexuel. La structure de ces glandes est parfois très bien décrite mais on sait peu de choses sur l'activité des substances qu'elles produisent.

D'autre part, quelques observations ont mis en évidence l'existence de phéromones sexuelles attractives, aphrodisiaques ou agrégatives chez quelques espèces, sans préciser les modalités de ces phénomènes.

Les perspectives de recherche sont donc nombreuses. En effet, il serait intéressant de préciser de multiples points :

- quel est le rôle des protéines souvent présentes en grandes quantités dans les sécrétions métathoraciques des adultes?

- la composition du contenu des glandes abdominales dorsales varie-t-elle au cours du développement post-embryonnaire et dans le cas où ces glandes persistent chez les adultes, produisent-elles les mêmes composés ?

- le contenu de ces mêmes formations dorsales estil semblable pour toutes les glandes d'une espèce donnée ? Si non, des différences fonctionnelles existentelles?

- quel est le rôle des nombreuses glandes présentant un dimorphisme sexuel (uradénies, glandes tégumentaires unicellulaires...) ?

- par quelles glandes sont produites et quelle est la nature chimique des quelques phéromones mises en évidence ? Ces phéromones existent-elles chez d'autres espèces ? 
Pour faire avancer les connaissances dans le domaine de la communication chimique chez les Hétéroptères, qui semble assez complexe et variée, l'accent devra être mis sur les études comportementales qui, en précisant l'activité biologique des molécules décrites, sont la clé d'une bonne compréhension des mécanismes mis en jeu.

\section{RÉFÉRENCES BIBLIOGRAPHIQUES}

Aldrich J. R., Yonke T. R., 1975. Natural products of abdominal and metathoracic scent glands of Coreoid bugs. Ann. entomol. Soc. Am., 68, 955-960.

Aldrich J. R., Blum M. S., Duffy S. S., 1976. Male-specific natural products in the bug, Leptoglossus phyllopus : chemistry and possible function. J. Insect Physiol., 22, 1201-1206.

Aldrich J. R., Blum M. S., Hefetz A., Fales H. M., Lloyd H. A., Roller P., 1978a. Proteins in a non-venomous defensive secretion: biosynthetic significance. Sci., 201, 452-453.

Aldrich J. R., Blum M. S., Lloyd H. A., Fales H. M., 1978b. Pentatomid natural products. Chemistry and morphology of the III-IV dorsal abdominal glands of adults. J. chem. Ecol., 4, 161-172.

Aldrich J. R., Blum M. S., Fales H. M., 1979a. Species-specific natural products of adult male leaf-footed bugs (Hemiptera : Heteroptera). J. chem. Ecol., 5, 53-62.

Aldrich J. R., Blum M. S., Lloyd H. A., Evans P. H., Burckhard D. R., 1979b. Novel exocrine secretions from two species of scentless plant bugs (Hemiptera: Rhopalidae). Entomol. exp. appl., 26, 323-331.

Aldrich J. R., Kochansky J. P., Lusby W. R., Dutky S. R., 1982. Volatile male-specific natural products of a Coreid bug (Hemiptera : Heteroptera). J. chem. Ecol., 8, 1369-1376.

Aldrich J. R., Lusby W. R., Kochansky J. P., Abrams C. B., 1984a. Volatile compounds from the predatory insect Podisus maculiventris (Hemiptera : Pentatomidae). J. chem. Ecol., 10, 561-568.

Aldrich J. R., Kochansky J. P., Abrams C. B., 1984b. Attractant for a beneficial insect and its parasitoid : pheromone of the predatory spined soldier bug, Podisus maculiventris (Hemiptera: Pentatomidae). Environ. Entomol., 13, 1301-1306.

Aldrich J. R., Oliver J. E., Lusby W. R., Kochansky J. P., 1986. Identification of male-specific exocrine secretions from predatory stink bugs (Hemiptera: Pentatomidae). Arch. Insect Biochem. Physiol., 3, 1-12.

Baker J. T., Kemball P. A., 1967. Volatile constituents of the scent gland reservoir of the Coreoid, Pternistria bispina Stal. Aust. J. Chem., 20, 395-398.

Baker J. T., Jones P. A., 1969. Volatile constituents of the scent gland reservoir of the nymph of the Coreoid, Pternistria bispina Stal. Aust. J. Chem., 22, 1793-1796.

Baker J. T., Blake J. D., Mac Leod J. K., Ironside D. A., Johnson I. C., 1972. The volatile constituents of the scent gland reservoir of the fruit-spotting bug, Amblypelta nitida. Aust. J. Chem., 25, 393-400.

Baldwin W. F., Knight A. G., Lynn K. R., 1971. A sex pheromone in the insect Rhodnius prolixus (Hemiptera: Reduviidae). Can. Entomol., 103, 18-22.

Blum M. S., Padovani F., Amante E., 1968. Alkanones and terpenes in the mandibular glands of Atta species (Hymenoptera : Formicidae). Comp. Biochem. Physiol., 26, 291-296.

Bohnag P. F., Wig J. R., 1953. The functional anatomy of the milkweed bug, Oncopeltus fasciatus (Dallas) (Heteroptera : Lygaeidae). J. Morphol., 93, 177-284.

Brennan B. M., Chang F., Mitchell W. C., 1977. Physiological effects of sex pheromone communication in the southern green stink bug, Nezara viridula (Heteroptera : Pentatomidae). Environ. Entomol., 6, 169-173.

Butenand A., Tam N., 1957. Uber einer geschlechtsspezifischen Duftstoff der Wasserwanze Belostoma indica Vitalis (Lethocerus indicus Lep.). Hoppe Seyler's Z. Physiol. Chem., 308, 277-283.

Calam D. H., Youdeowi A., 1968. Identification and functions of secretion from the posterior scent gland of fifth instar larva of the bug Dysdercus intermedius. J. Insect Physiol., 14, 1147-1158.
Calam D. H., Scott G. C., 1969. The scent gland complex of the adult cotton stainer bug, Dysdercus intermedius. J. Insect Physiol., $15,1695-1712$.

Carayon J., 1948. Dimorphisme sexuel des glandes odorantes métathoraciques chez certains Hémiptères. C. R. Sc. Acad. Sci. Fr., 227, 303-305.

Carayon J., 1954. Un type nouveau d'appareil glandulaire propre aux mâles de certains Hémiptères Anthocorides. Bull. Mus.. $2^{\mathrm{e}} \mathrm{s}$., XXVI, 602-606.

Carayon J., 1971. Notes et documents sur l'appareil odorant métathoracique des Hémiptères. Ann. Soc. entomol. Fr., 7, 737-770.

Carayon J., 1981. Dimorphisme sexuel des glandes tégumentaires et production de phéromones chez les Hémiptères Pentatomoidea. C. R. Sc. Acad. Sci. Paris (ser. III), 29, 867-870.

Carayon J., 1984. Les androconies de certains Hémiptères Scutelleridae. Ann. Soc. entomol. Fr., 20, 113-134.

Collins R. P., Drake T. H., 1965. Carbonyl compounds produced by the meadow plant bug, Leptoterna dolabrata (Hemiptera : Miridae). Ann. entomol. Soc. Am., 58, 764-765.

Eisner T., Johnesse J. S., Carre J., Hendry L. B., Meinwald J., 1971. Defensive use by an insect of a plant resin. Science, 184, 996999.

Everton I. J., Staddon B. W., 1979. The accessory gland function in Oncopeltus fasciatus. J. Insect Physiol., 25, 133-141.

Everton I. J., Games D. E., Staddon B. W., 1974. Composition of the scent from Apodiphus amygdali. Ann. entomol. Soc. Am., 67, 815-816.

Everton I. J., Knight D. W., Staddon B. W., 1979. Linalool from the metathoracic scent gland of the cotton stainer Dysdercus intermedius Distant (Heteroptera: Pyrrhocoridae). Comp. Biochem. Physiol., 63B, 157-161.

Filshie B. K., Waterhouse D. F., 1969. The fine structure of the lateral scent-glands of the green vegetable bug, Nezara viridula. Tissue \& Cell, 366-385.

Games D. E., Staddon B. W., 1973a. Composition of scents from the larva of the milkweed bug Oncopeltus fasciatus. J. Insect Physiol., 19, 1527-1532.

Games D. E., Staddon B. W., 1973b. Chemical expression of a sexual dimorphism in the tubular scent-gland of the milkweed bug Oncopeltus fasciatus. Experientia, 29, 532-533.

Gilby A. R., Waterhouse D. F., 1967. Secretions from the lateral scent glands of the green vegetable bug, Nezara viridula. Nature, 216, $90-91$.

Gough A. J. E., Hamilton J. G. C., Games D. E., Staddon B. W., 1985. Multichemical defense of plant bug Hotea gambiae (Westwood) (Heteroptera : Scrutelleridae). J. chem. Ecol., 11, 342-352.

Hanssen H. P., Jacob J., 1982. Monoterpenes from the true bug Harpocera thoracica (Hemiptera). Z. Naturforsch., 37e, 1281-1282.

Harris V. E., Todd J. W., 1980. Male-mediated aggregation of male, female and fifth instar southern green stink bug and concomitant attraction of a Tachinid parasite, Trichopoda pennipes. Ent. exp. appl., 27, 117-126.

Harris V. E., Todd J. W., Webb J. C., Benner J. C., 1982. Accoustical and behavioral analysis of the songs of the southern green stink bug, Nezara viridula. Ann. entomol. Soc. Am., 75, 234-249.

Honda K., 1981. Larval osmetrial secretions of the swallowtails (Papilio). J. chem. Ecol., 7, 1089-1113.

Hubell S. P., Wiemer D. F., Adejare A., 1983. An antifungal terpenoid defends a neotropical tree against attacks by fungus growing ants (Atta). Oecologia (Berlin), 60, 321-327. 
Ishiwatari T., 1974. Studies of the scent of stink bugs. I. Alarm pheromone activity. Appl. Entomol. Zool., 9, 153-158.

Ishiwatari T., 1976. Studies of the scent of stink bugs. II. Aggregation pheromone activity. Appl. Entomol. Zool., 11, 38-44.

King A. B. S., 1983. Studies of sex attraction in the cocoa Capsid Distantiella theobroma (Heteroptera: Miridae). Entomol. exp. appl., 16, 243-254.

Kitamura C., Wakamura S., Takahashi S., 1984. Identification and functions of ventral glands secretion of some Heteroptera. Appl. Entomol. Zool., 19, 33-41.

Knight D. W., Rossiter M., Staddon B. W., 1984. (Z,E)- $\alpha-$ farnesene : major component of secretion from metathoracic scent gland of cotton stainer seed bug Oxycarenus hyalinipennis (Costa) (Heteroptera : Lygaeidae). J. chem. Ecol., 10, 641-649.

Lawrence P. A., Staddon B. W., 1975. Peculiarities of the epidermal gland system of the cotton stainer Dysdercus fasciatus (Signoret) (Heteroptera : Pyrrhocoridae). J. Entomol., 49, 121-130.

Levinson H. Z., Levinson A. R., Muller B., Steinbrecht R. A., 1974. Structure of sensilla, olfactory perception and behavior of the bedbug Cimex lectularius, in response to its alarm pheromone. $J$. Insect Physiol., 20, 1231-1248.

Mac Leod J. K., Horve I., Cable J., Blake J. D., Baker J. T., Smith D., 1975. Volatile scent gland components of some tropical Heteroptera. J. Insect Physiol., 21, 1219-1224.

Mitchell W. C., Mau R. F. L., 1971. Response of the female southern green stink bug and its parasite, Trichopoda pennipes, to male stink bug pheromone. J. econ. Entomol., 64, 856-859.

Moore B. P., 1968. Studies on the chemical composition and function of the cephalic gland secretion in Australian termites. J. Insect Physiol., 14, 33-39.

Moriya S., Shiga M., 1984. Attraction of the male brown-winged green bug Plautia stali Scott (Heteroptera : Pentatomidae) for males and females of the same species. Appl. Entomol. Zool., 19, 317-322.

Olagbemiro T. O., Staddon B. W., 1983. Isoprenoids from metathoracic scent gland of cotton seed bug, Oxycarenus hyalinipennis (Costa) (Heteroptera : Lygaeidae). J. chem. Ecol., 9, 1397-1412.

Pattendon G., Staddon B. W., 1968. Secretion of the metathoracic gland of the waterbug Notonecta glauca L. (Heteroptera : Notonectidae). Experientia, 24, 1092.

Pavis C., Malosse C., 1986. Mise en évidence d'un attractif sexuel produit par les mâles de Nezara viridula (L.) (Heteroptera : Pentatomidae). C. R. Acad. Sci. Paris, Ser. III, 7, 273-276.
Percy J., Mac Donald J. A., Weatherston J., 1980. Ultrastructure of scent glands in larvae of Apateticus bracteatus and chemical composition of the secretion. Can. J. Zool., 58, 2105-2115.

Prestwich G. D., 1976. Composition of the scents of eight East African Hemipterans. Nymph-adult chemical polymorphism in Coreids. Ann. entomol. Soc. Am., 69, 812-814.

Remold H., 1962. Über die biologische Bedeutung der Duftdrüsen bei den Landwanze (Geocorisae). Z. vergl. Physiol., 45, 633-694.

Remold H., 1963. Scent glands of land bugs, their physiology and biological functions. Nature, 198, 764-768.

Roth L. M., 1961. A study of the odoriferous glands of Scaptocoris divergens (Hemiptera : Cydnidae). Ann. entomol. Soc. Am., 54, 900-901.

Scales A. L., 1968. Female tarnished plant bugs attract males. $J$. econ. Entomol., 61, 1466-1467.

Staddon B. W., 1971. Metasternal scent glands in Belostomatidae (Heteroptera). J. Entomol. (A), 46, 69-71.

Staddon B. W., 1979. The scent glands of Heteroptera. Adv. Insect Physiol., 14, 351-419.

Staddon B. W., Everton I. J., Games D. E., 1979. Organ specificity and scent constitution in Corixidae (Heteroptera: Hydrocorisidae). Comp. Biochem. Physiol., 62B, 259-262.

Strong F. E., Shelgal J. A., Hughes P. R., Hussein E. K. M., 1970. Reproductive biology of Lygus hesperus Knight. Hilgardia, 40, 105143.

Thouvenin M., 1965. Etude préliminaire des « uradénies » chez certains Hémiptères Pentatomorphes. Ann. Soc. entomol. Fr., 1, 973998.

Vrkoc J., Ubik K., Zdarek J., Kontev C., 1977. Ethyl acrylate and vanillin as components of the male sex-pheromone complex in Eurygaster integriceps. Acta Entomol. Bohemoslov., 74, 205-206.

Waterhouse D. F., Gilby A. R., 1964. The adult scent gland of nine bugs of the superfamily Coreoidea. J. Insect Physiol., 10, 977-987.

Waterhouse D. F., Forss D. A., Hackman R. H., 1961. Characteristic odour components of the stink bugs. I. Insect Physiol., 6, 113121

Youdeowi A., Calam D. H., 1969. The morphology of the scent gland of Dysdercus intermedius Distant (Hemiptera : Pyrrhocoridae) and preliminary analysis of the scent gland secretions of the fifth instar larvae. Proc. R. entomol. Lond. (A), 44, 38-44. 